\section{ACCUMULATOR RING LATTICE FOR THE NATIONAL SPALLATION NEUTRON SOURCE}

\author{
C.J. Gardner*, Y.Y. Lee and A.U. Luccio \\ Brookhaven National Laboratory, Upton, NY 11973.
}

RECEIVED

JUN 25 997

OSTI

\section{Abstract}

The Accumulator Ring for the proposed National Spallation Neutron Source (NSNS) [1] is to accept a 1.03 millisecond beam pulse from a $1 \mathrm{GeV}$ Proton Linac at a repetition rate of $60 \mathrm{~Hz}$. For each beam pulse, $10^{14}$ protons are to be accumulated via charge-exchange injection. A 295 nanosecond gap in the beam, maintained by an if system, will allow for extraction to an external target for the production of neutrons by spallation. This paper describes the four-fold symmetric lattice that has been chosen for the ring. The lattice contains four long dispersion-free straight sections to accomodate injection, extraction, if cavities, and beam scraping respectively. The four-fold symmetry allows for easy adjustment of the tunes and flexibility in the placement of correction elements, and ensures that potentially dangerous betatron structure resonances are avoided.

\section{THE LATTICE}

The lattice for the accumulator ring will consist of four superperiods, each containing a $90^{\circ}$ arc and a long straight section [2]. The layout of the accumulator is shown in Figure 1. Each arc will consist of four identical FODO

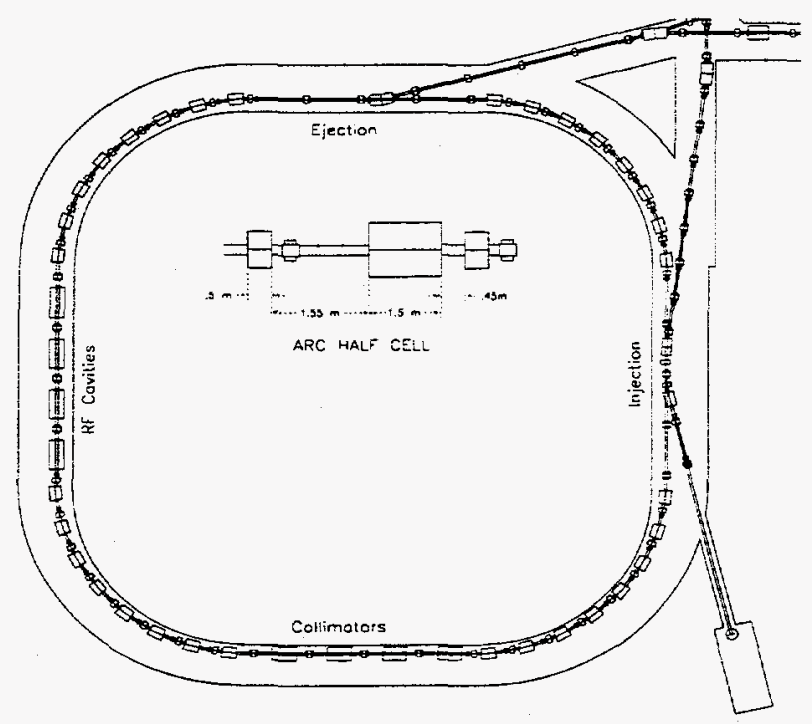

Figure 1: Accumulator ring layout.

cells and will have a total betatron phase advance of $2 \pi$ which will ensure zero dispersion in the long straight sections. The arc half-cells will be 4 meters long and consist

\footnotetext{
* Work sponsored by the Division of Material Sciences, U.S. Department of Energy, under contract DE-AC05-96OR22464 with Lockheed Martin Energy Research Corp, for Oak Ridge National Laboratory.
}

Table 1: NSNS Accumulator ring parameter
\begin{tabular}{|cc|}
\hline Kinetic Energy & $1.0 \mathrm{GeV}$ \\
Magnetic Rigidity & $5.6575 \mathrm{~T}-\mathrm{m}$ \\
Circumference & $220.688 \mathrm{~m}$ \\
Periodicity & 4 \\
Structure & $24 \mathrm{FODO}$ \\
Beam Emittance & $120 \pi \mathrm{mm}-\mathrm{mrad}$ \\
Ring Admittance & $312 \pi \mathrm{mm}-\mathrm{mrad}$ \\
$\beta_{\max }(\mathrm{x} / \mathrm{y})$ & $19.2 / 19.2$ \\
$X_{p}(\mathrm{max} / \mathrm{min})$ & $4.1 / 0.0 \mathrm{~m}$ \\
$\nu_{x / y}$ & $5.82 / 5.80$ \\
Natural $\xi_{x / y}$ & $-6.6 /-7.3$ \\
$\gamma_{t}$ & 4.93 \\
\hline
\end{tabular}

of a half-quadrupole, a $1.55 \mathrm{~m}$ space, a $1.5 \mathrm{~m}$ long dipole, a $0.45 \mathrm{~m}$ space, and a half-quadrupole. Two identical FODO cells without a dipole will form each long straight section. Here the half-cells will be 5.793 meters long and the phase advances will be adjusted so that the horizontal and vertical tunes of the ring are 5.82 and 5.8 respectively. The lattice functions for one superperiod are shown in Figure 2 where the plot runs from the center of one long straight section to the next. The relevant ring parameters are given in Table 1.

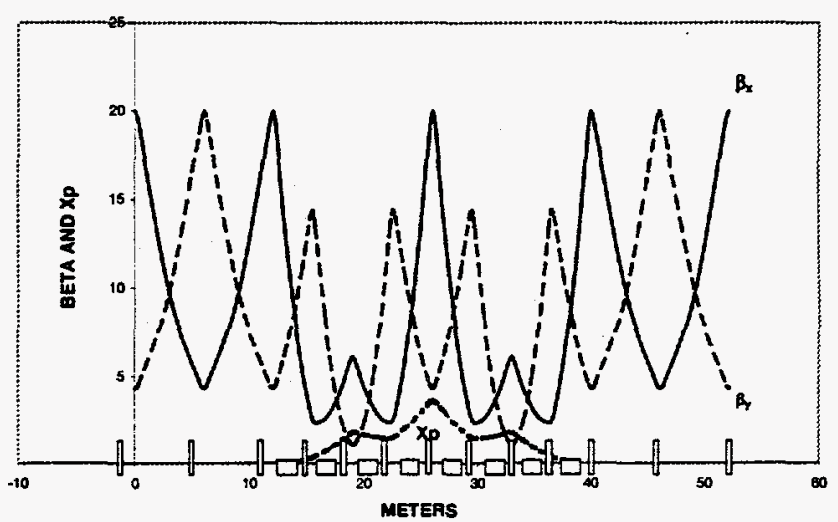

Figure 2: Accumulator lattice functions.

The four superperiods of the ring are labeled A, B, C, and $D$, and run sequentially along the beam direction from the beginning of one arc to the next. The order of magnets in each superperiod X is DHX1, QVX1, DHX2, QHX2, ..., DHX8, QHX8, QVX9, QHX10, QVX11, and QHX12, where $\mathrm{D}$ and $\mathrm{Q}$ denote dipoles and quadrupoles and $\mathrm{H}$ and $\mathrm{V}$ refer to the horizontal and vertical planes. The long 
Table 2: Tunability and Chromaticity Behavior.

\begin{tabular}{|c|c|c|c|c|c|}
\hline$\Delta p / p$ & $\Delta \nu_{x}$ & $\Delta \nu_{y}$ & $\beta_{x, \max }$ & $X_{p, \max }$ & $\Delta F Q$ \\
$\%$ & & & $\begin{array}{c}\beta_{y, \max } \\
X_{p, \min }\end{array}$ & $\begin{array}{c}\Delta D Q \\
{[\mathrm{~m}]}\end{array}$ \\
\hline 0.0 & 0.0 & 0.0 & 19.2 & 4.1 & 0.0 \\
& & & 19.2 & 0.0 & 0.0 \\
\hline 0.0 & -0.5 & 0.0 & 21.9 & 4.1 & -18.1 \\
& & & 17.8 & 0.0 & -4.3 \\
\hline 0.0 & 0.0 & -0.5 & 17.8 & 4.1 & -4.3 \\
& & & 21.9 & 0.0 & -18.1 \\
\hline 0.0 & -0.5 & -0.5 & 20.1 & 4.1 & -24.8 \\
& & & 20.1 & 0.0 & -24.8 \\
\hline \hline 0.5 & -0.03 & -0.04 & 19.9 & 4.16 & \\
\hline 1.0 & -0.06 & -0.07 & 20.5 & 4.23 & \\
\hline
\end{tabular}

straight section in A runs from QVA9 through QHA12 and will be used for injection. Similarly, the long straight sections in B, C, D will be used for beam scraping collimators, If cavities, and extraction respectively. In the arcs, the $\mathbf{1 . 5 5}$ $\mathrm{m}$ space between a quadrupole and the downstream dipole will contain a beam position monitor (pickup electrode) followed by a correction package and a vacuum pumping port. The correction package will consist of a dipole corrector and either a skew quadrupole or higher-order multipole corrector. Position monitors and correction packages will also be located downstream of each quadrupole in the long straight sections. Other available space in the lattice will be used to accommodate tune meter kickers, a transverse damping system, and clearing electrodes, to name a few.

The beam will have a full emittance, both horizontal and vertical, of $120 \pi \mathrm{mm}$-mrad and the ring will have a full acceptance of $312 \pi \mathrm{mm}$-mrad, which is twice the full beam emittance plus an allowance of $1 \mathrm{~cm}$ for the maximum possible orbit errors. The ring must also accept a maximum beam momentum spread of $\pm 1 \%$ and the quadrupoles QHX4 at the centers of the arcs will be $50 \%$ larger than the others to meet this requirement. The quadrupoles $\mathrm{QHX10}$ in the centers of the long straights will also have larger apertures to allow for the injection orbit bumps and for the extraction kicker and septum displacements.

\section{TUNABILITY AND CHROMATIC BEHAVIOR}

It will be desirable for the accumulator ring to run with different sets of betatron tunes. Small tune changes are expected in normal ring operations, however tune changes of up to half a unit may be implemented in special operations for accelerator studies. Table 2 shows the behavior of the beta function for a tune change of 0.5 units. As can be seen in the Table, a change in tune of half a unit results in a change of less than $15 \%$ in the beta function, with no change in the dispersion function. Because the chromatic behavior is nonlinear, the betatron functions for an off- momentum particle might show significant changes, but for this lattice, even at $1 \%$ off-momentum, the maximum ever expected, the betatron functions and the dispersion behave very well.

In practice the desired tunability will be achieved by dividing the main windings of the quadrupoles into three families, with the magnets of each family connected in series to a separate power supply. The first family will consist of all 28 arc quadrupoles (QVX1, QHX2, .., QVX7); the second will consist of all 12 focusing quadrupoles $(\mathrm{QHX} 8$, $\mathrm{QHX10}$ and $\mathrm{QHX12}$ ) in the long straight sections; and the third will consist of all 8 defocusing quadrupoles (QVX9 and QVX11) in the long straights. The current in the first family will be adjusted so that the phase advance of each $\operatorname{arc}$ is $2 \pi$, thereby ensuring zero dispersion in the long straight sections. The currents in the second and third families will then be used to adjust the tunes. The quadrupoles will also have trim windings which will be divided into four families. These will be used for fine tuning the lattice. In particular they will allow for independent adjustment of the currents in the quadrupoles at the center (QHX10) and the ends (QHX8 and QHX12) of the long straight sections. This is important in the A superperiod, for example, where the injection setup depends on the setting of the QHA10 quadrupole.

\section{CORRECTION ELEMENTS}

To compensate for unavoidable field imperfections and magnet alignment errors, the accumulator ring will have a set of correction elements consisting of horizontal and vertical dipoles, skew quadrupoles, sextupoles, skew sextupoles, and octupoles. Trim windings on the lattice quadrupoles will allow for any necessary quadrupole corrections. The correction elements will be contained in packages located downstream of the position monitor at each lattice quadrupole. Each package, except those downstream of the quadrupoles QHX10 (at the centers of the long straight sections), will contain a horizontal or vertical dipole and either a skew quadrupole or higher-order multipole. The packages downstream of quadrupoles QHX10 will contain only horizontal dipoles. Thus, there will be a total of 24 horizontal and 24 vertical dipoles. Each superperiod will have four skew quadrupoles (QSX1, QSX3, QSX5, QSX7) located in the correction packages downstream of quadrupoles QVX1, QVX3, QVX5, and QVX7; two sextupoles (SHX8, SVX9) located downstream of QHX8 and QVX9; two skew sextupoles (SSVX11, SSHX12) located downstream of QVX11 and QHX12; and three octupoles (OHX2, OHX4, OHX6) located downstream of $\mathrm{QHX} 2, \mathrm{QHX} 4$, and $\mathrm{QHX} 6$.

Closed orbit correction is discussed in Ref. [3].

\section{RESONANCE CORRECTION}

Because the ring will operate at very high intensities for which stringent limits on losses will be imposed, the possibility of beam loss due to resonance excitation must be 
considered. The second, third and fourth-order resonances between tunes of 5 and 6 are shown in Figure 3. Although the space-charge tune spread of the beam is expected to be small $(\sim 0.1)$, there are a number of resonance lines sufficiently close to the working point to be of concern. Moreover, even though lines such as those excited by normal and skew quadrupoles are far from the working point, they can cause unfavorable distortions of the betatron functions. The four-fold symmetric lattice allows placement of correction elements so that either even or odd harmonics (in azimuth $\theta$ ) can be produced for the correction of the following resonances. Detailed calculations are underway to determine the required magnet strengths.

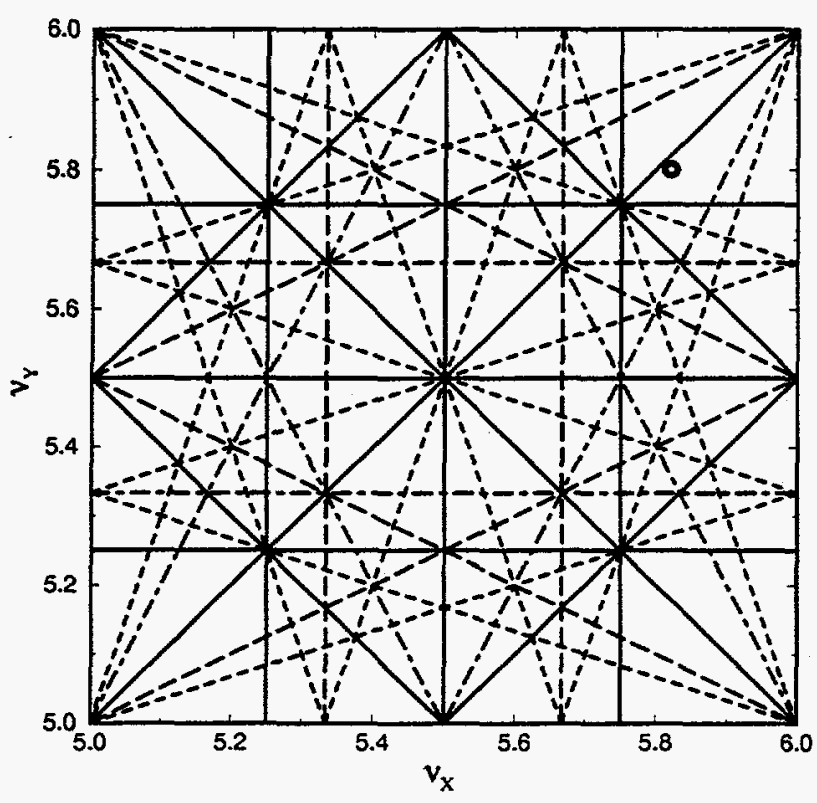

Figure 3: Accumulator Tune Chart. The circle shows the working point. Solid and short-dashed lines indicate second and fourth-order resonances; long-dashed and dotdashed lines indicate third-order.

\subsection{Second-Order Resonances}

The $2 \nu_{x}=11$ and $2 \nu_{y}=11$ resonances can be corrected by exciting the trim windings on the quadrupoles with harmonic 11 in azimuth $\theta$. (To produce an odd harmonic, magnets separated by $\theta=180^{\circ}$ are excited with equal but opposite currents.) Similarly, the sum resonance $\nu_{x}+\nu_{y}=11$ can be corrected by exciting the skew quadrupole correctors with harmonic 11 ; the difference resonance $\nu_{x}-\nu_{y}=0$ can be corrected (or enhanced if coupling between the two planes is desired) by exciting these correctors with harmonic 0 .

\subsection{Third-Order Resonances}

The $3 \nu_{x}=17$ and $\nu_{x}+2 \nu_{y}=17$ resonances can be corrected by exciting sextupoles with harmonic 17 . The eight sextupole correctors allow for independent correction of the two lines. Similarly, the $3 \nu_{y}=17$ and $\nu_{y}+2 \nu_{x}=17$ resonances can be corrected with the eight skew sextupole correctors.

\subsection{Fourth-Order Resonances}

The fourth-order resonance lines are driven by octupoles (both normal and skew) and those closest to the working point pass through the point $\nu_{x}=\nu_{y}=5.75$ in Figure 3 . The effects of these lines can be reduced by exciting the octupole correctors with harmonic 0 . Schemes for correcting specific lines are under investigation. The octupole correctors also allow for Landau damping of transverse instabilities.

\section{STRUCTURE RESONANCES}

One consideration in our choice of a lattice was the avoidance of structure resonances. The 4-fold symmetric lattice has fourth-order structure resonances at betatron tunes of 5 and 6; third-order at tunes of 5.333 and 6.666; and second-order at a tune of 6 . However, none of these are near the proposed working point. A 3-fold symmetric lattice that has been considered in previous design studies, on the other hand, has fourth-order structure resonances at a betatron tune of 3.75 which is right on top of the proposed working point for this lattice. Although these resonances may not hurt the ring performance, they are best avoided.

\section{REFERENCES}

[1] W.T. Weng, et. al., "Accumulator Ring Design for the NSNS Project", these proceedings.

[2] Y.Y. Lee, "The 4 Fold Symmetric Lattice for the NSNS Accumulator Ring", BNLNSNS Tech.Note No. 026, February 14, 1997.

[3] C.J. Gardner, "Closed Orbit Distortion and Correction in the Four-Fold Symmetric NSNS Ring", BNLNSNS Tech.Note No. 031, April 14, 1997. 


\section{DISCLAIMER}

This report was prepared as an account of work sponsored by an agency of the United States Government. Neither the United States Government nor any agency thereof, nor any of their employees, make any warranty, express or implied, or assumes any legal liability or responsibility for the accuracy, completeness, or usefulness of any information, apparatus, product, or process disclosed, or represents that its use would not infringe privately owned rights. Reference herein to any specific commercial product, process, or service by trade name, trademark, manufacturer, or otherwise does not necessarily constitute or imply its endorsement, recommendation, or favoring by the United States Government or any agency thereof. The views and opinions of authors expressed herein do not necessarily state or reflect those of the United States Government or any agency thereof. 


\section{DISCLAIMER}

Portions of this document may be illegible in electronic image products. Images are produced from the best available original document. 